\title{
Periphere Stimulation
}

\author{
Hubert R. Dinse
}

\begin{abstract}
Neben direkter zentralnervöser Stimulation wie TMS oder tDCS und roboter- und gerätegestützter Rehabilitation wurden Therapieansätze basierend auf peripherer Stimulation entwickelt. Die bisher an Gesunden erhobenen Befunde deuten darauf hin, dass die repetitive sensorische Stimulation zu einer weitreichenden Reorganisation in den sensomotorischen Netzwerken führt. Die bisher publizierten Metastudien verweisen darauf, dass die alleinige oder in Kombination mit anderen Therapiemaßnahmen erfolgende Anwendung der peripheren Stimulation zu einem verbesserten Rehabilitationserfolg führt. Die Möglichkeit, die Stimulation zu Hause über lange Zeit anzuwenden zu können, dürfte gerade für die Langzeitbehandlung chronischer Patienten eine wichtige Rolle spielen.
\end{abstract}

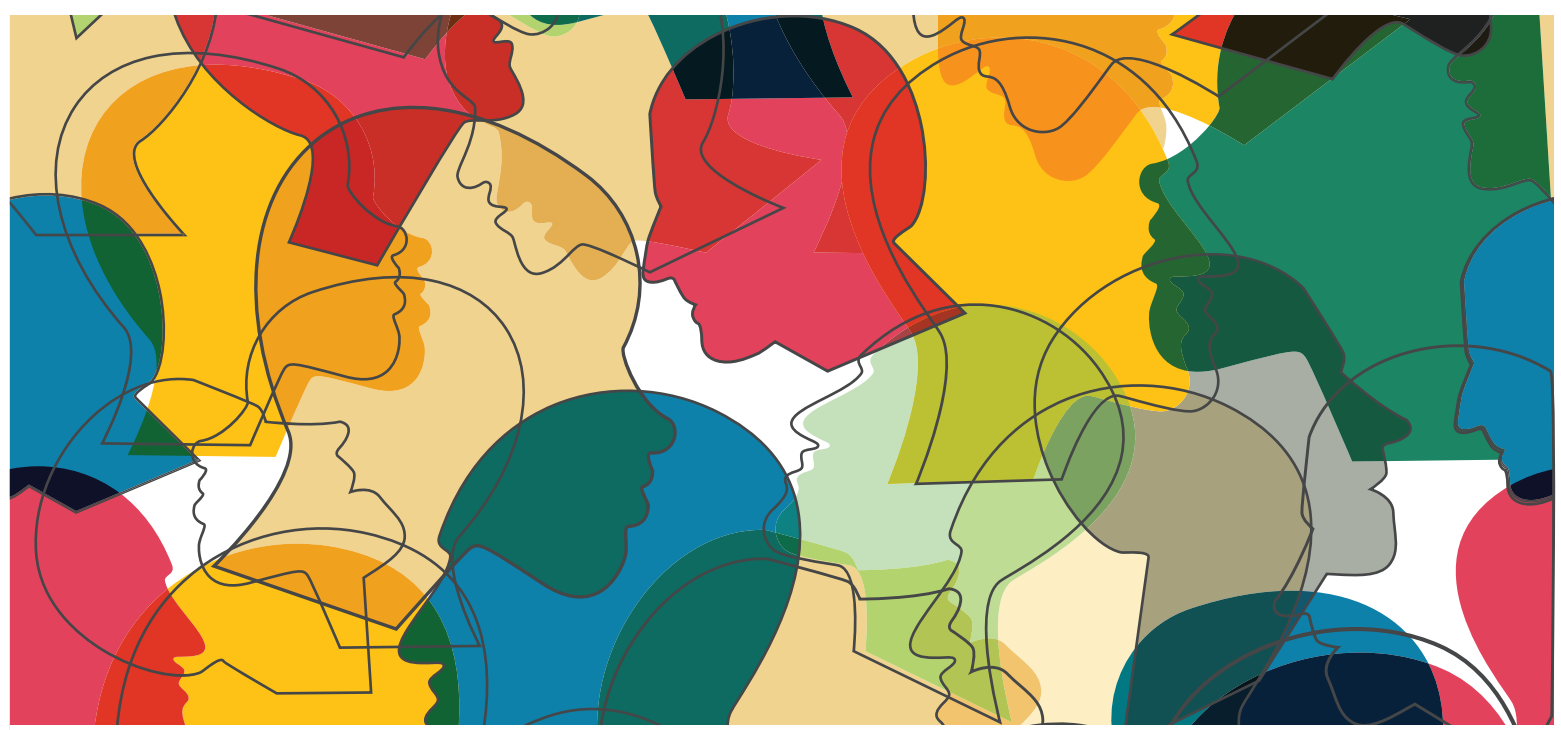

Die bisherigen Erfahrungen mit peripherer Stimulation der oberen Extremität verweisen auf deutliche Verbesserungen des Tastsinns, der Propriozeption, der haptischen und motorischen Performanz, was sich in einer erhöhten Alltagskompetenz niederschlägt. (Quelle: kubko/stock.adobe.com)

\section{Einleitung}

Die nach einem Schlaganfall auftretenden massiven sensomotorischen Beeinträchtigungen haben trotz vorhandener rehabilitativer Behandlungen weitreichende physische, psychologische, finanzielle und soziale Auswirkungen. Beeinträchtigungen der Willkürmotorik und der Somatosensorik gehören zu den häufigsten Folgen des Schlaganfalls, wobei die Auswirkungen auf obere und untere Extremitäten vergleichbar sind. Es herrscht heute Einigkeit darüber, dass die Unversehrtheit des somatosensorischen Inputs nicht nur für die taktile Wahrnehmung, sondern auch für die sensomotorische Leistung von entscheidender Bedeutung ist. Der Verlust sensorischer Fähigkeiten der obe- ren Extremitäten, insbesondere der Hand, verstärkt, trotz möglicher Erholung motorischer Funktionen, die Komplikationen bei Nutzung der Hand für allgemeine Alltagstätigkeiten [3].

Therapeutische Intervention und Leistungssteigerung Grundsätzlich nutzen alle auf neuroplastischen Prinzipien basierenden Rehabilitationsmaßnahmen nach Schlaganfällen aufgabenspezifisches Training in Verbindung mit erheblichem Übungsaufwand, um Plastizitätsprozesse auszulösen und damit sensomotorische Funktionen zu verbessern [26]. Da viele Patienten an eingeschränkter Mobilität leiden, sind diese Therapieansätze 
aus oben genannten Gründen oft nur bedingt zu realisieren. Demzufolge werden die bisherigen Erkenntnisse zur Effektivität von Standardtherapieverfahren im Rahmen der Leistungswiederherstellung von Patienten kontrovers diskutiert. Insbesondere für die oberen Extremitäten konnte bislang nur ein bedingter Zusammenhang zwischen dem Umfang therapeutischer Intervention und der daraus resultierenden Leistungssteigerung festgestellt werden [17]. Nach einer 2014 veröffentlichten Metastudie gab es zum genannten Zeitraum keine „,high-quality evidence“ für Interventionsmaßnahmen, die im Rahmen der Routineversorgung von Schlaganfallpatienten im Einsatz sind [22]. Aus diesem Grund ist die Entwicklung zusätzlicher Ansätze notwendig, die konventionelle Trainingsprozeduren ergänzen und verstärken.

Neue Rehabilitationsmaßnahmen In den letzten Jahren wurden Anstrengungen unternommen, die Wirksamkeit und Realisierbarkeit neuartiger Rehabilitationsmaßnahmen auf ihre Effizienz hin zu untersuchen. Neben direkter zentralnervöser Stimulation wie TMS oder tDCS und roboter- und gerätegestützter Rehabilitation wurden Ansätze mithilfe peripherer Stimulation untersucht.

\section{Repetitive sensorische Stimulation in der Rehabilitation}

Das Konzept sensorischer Stimulation zur Auslösung von Plastizitätsprozessen wird von verschiedenen Labors untersucht, die unterschiedliche Motivationen und Begriffe nutzen wie beispielsweise „peripheral nerve stimulation“ [24], „somatosensory stimulation“ [5][27], „unattended-based learning“ [9] oder „high-frequency stimulation“ [23]. Das Prinzip der „co-activation“ unterstreicht die Bedeutung des Hebb'schen Lernens, wonach im Gehirn synchrone neuronale Aktivität eine wichtige Voraussetzung zur Auslösung plastischer Veränderungen ist [7]. Manche Labore nutzen „stimulus-selective response plasticity“ oder „tetanic stimulation“ in Anlehnung an das Konzept der tetanischen Stimulation im Rahmen synaptischer Plastizitätsuntersuchungen [4]. Der häufig verwendete Begriff „passive Stimulation“ oder „passives Lernen“ soll deutlich machen, dass zeitlich strukturierte sensorische Reize verwendet werden, ohne diese aktiv zu beachten oder beachten zu müssen. Im Rahmen dieses Beitrags wird durchgehend der Begriff „repetitive sensorische Stimulation“ benutzt.

Elektrische Stimulationsansätze Seit geraumer Zeit kommt bereits eine Reihe von elektrischen Stimulationsansätzen zum Einsatz, bei denen die Unterstützung der motorischen Performanz durch Intensivierung sensorischen Inputs im Vordergrund steht. Dabei unterscheidet man die funktionelle (FES) und die therapeutische Elektrostimulation (TES) sowie nach ihren technischen und konzeptionellen Charakteristika die neuromuskuläre Elektrostimulation, EMG-getriggerte Elektrostimulati- on, Positions-Feedback-Stimulationstraining oder transkutane elektrische Nervenstimulation. Zu dem Problem einer einheitlich-verbindlichen Terminologie kommt eine noch größere Unübersichtlichkeit an Applikationsverfahren und Stimulationsparametern. Nur bezogen auf die Behandlung der oberen Extremität wird beispielsweise der Medianusnerv stimuliert, ein einzelner Finger (Fingerspitze) oder alle fünf Finger einer Hand (tipstim ${ }^{\circledR}$ ) oder die ganze Hand (mesh glove). Als Elektroden kommen Klebeelektroden, Ag-AgCl-Elektroden oder Handschuhe zum Einsatz („mesh-glove“, tipstim ${ }^{\circledR}$-Handschuh).

Geringe Vergleichbarkeit Besonders schwer wird eine vergleichende Bewertung bereits publizierter Arbeiten durch die Verwendung ganz unterschiedlicher Stimulationsparameter, die sowohl kontinuierliche als auch intermittierende Stimulationsprotokolle im Bereich zwischen $1 \mathrm{~Hz}$ und $100 \mathrm{~Hz}$ umfassen. Variabel ist auch die Dauer einer Stimulationssession, die zwischen Minuten bis mehrere Stunden betragen kann. Während einige Studien lediglich auf einer Stimulationssession beruhen, nutzen andere Untersuchungen eine wiederholte Applikation über mehrere Wochen bis hin zu vielen Monaten. Auch hinsichtlich des Versuchsdesigns gibt es Unterschiede. So kann repetitive Stimulation als „Stand-alone“-Verfahren genutzt werden oder in Kombination mit Training und anderen Therapiemaßnahmen. Schließlich kommen sehr unterschiedliche Arten des Assessments des Interventionsoutcomes zum Einsatz, wie z. B. die Verwendung von klinischen „rating scales“, von einfachen Funktionstests bis zu aufwendigen Verhaltenstests. Aufgrund dieser Vielfalt ist die Aussagekraft von Metaanalysen eingeschränkt [6].

\section{Konzeptioneller Hintergrund der Verwendung repetitiver Stimulation}

Der Königsweg zur Verbesserung sensorischer, motorischer oder kognitiver Leistungen besteht in lang andauerndem Training und Übung. Neuere Studien an Gesunden zeigen jedoch, dass vergleichbare Leistungsverbesserung auch ohne Training durch rein passive Darbietung sensorischer Reize erzielt werden kann. Solches „trainingsunabhängiges Lernen “ löst nachhaltige Veränderungen der Wahrnehmung und der neuronalen Verarbeitung aus.

Synaptische Plastizität Die Grundlage aller Lernvorgänge sind Veränderungen in der Kommunikation zwischen Nervenzellen. Auf zellulärer Ebene erfolgt der Lernprozess, indem die Signalübermittlung an der Synapse effizienter oder weniger effizient wird [1][19]. Diese Modifikation in der Synapsenstärke bezeichnet man als synaptische Plastizität. Eine Reihe von Modellen beschreibt, wie sich die synaptische Übertragung im Verlauf des Lernens nachhaltig ändert, z. B. durch Langzeitpotenzierung (LTP) und Langzeitdepression (LTD). So löst hochfrequente elektrische Stimulation von Nervenzellen LTP aus, das heißt, 


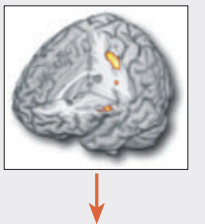

Modifikation der synaptischen Effizienz

LTP-artige Prozesse

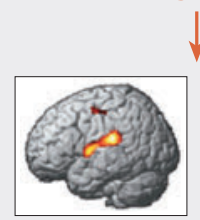

prä

$\downarrow$

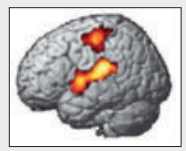

post

kortikale Reorganisation

Umbau taktiler und sensomotorischer Verarbeitung

Rekruitment kortikaler

Verarbeitungsressourcen

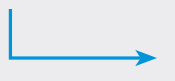

okalisierte repetitive Aktivierung in der Fingerrepräsentation

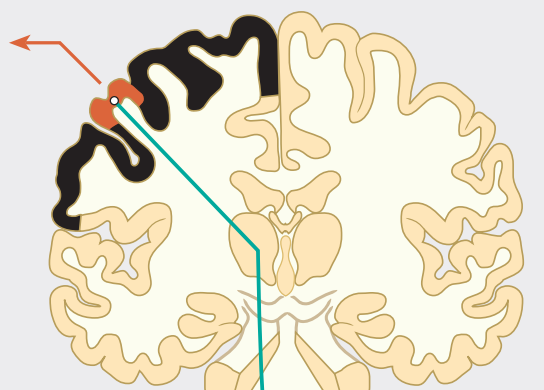

LTP-artige

repetitive sensorische

Stimulation

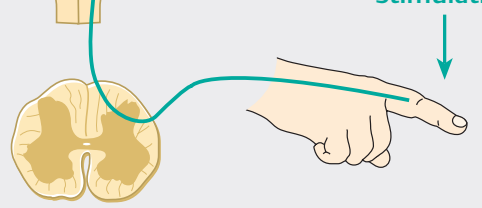

Reorganisation der taktilen und sensomotorischen Verarbeitung

Verbesserung taktil/haptischer Wahrnehmung und sensomotorischer Leistungen/Funktionsrestauration

-Abb. 1 Wirkungsschema der repetitiven Stimulation. Sensorische Stimulation der Finger mittels LTP-artigen Stimulationsprotokollen löst eine Kaskade von funktionellen Veränderungen des somatosensorischen Systems aus. Im Mittelpunkt steht dabei die Annahme, dass die Art der sensorischen Stimulation plastische Prozesse induziert, die ihrerseits zu Verhaltensänderungen führen, im Falle von Patienten zu einer Funktionsrestauration der oberen Extremität. BOLD-Aktivierungen gemessen während bzw. vor und nach der Stimulation. (Quelle: nach Dinse u. Tegenthoff [10]; Umsetzung: Thieme Gruppe).

die Kommunikation zwischen den stimulierten Zellen verstärkt sich. Niederfrequente Stimulation verursacht hingegen LTD; die Effizienz der Kommunikation zwischen den Zellen nimmt ab. LTD und LTP sind somit zwei zentrale Mechanismen für Lernprozesse. Die Wirksamkeit der repetitiven Stimulation beruht wahrscheinlich darauf, dass die verwendeten Stimulationsprotokolle zur Auslösung synaptischer Plastizität nahezu optimal sind.

\section{Wirkung repetitiver Stimulation bei Gesunden}

Die Kenntnis der mutmaßlichen Mechanismen, die im intakten Gehirn zu Plastizitätsprozessen und einhergehenden Verhaltensänderungen führen, sind eine, wenn auch nicht die entscheidende Voraussetzung für den Einsatz als Intervention bei Patienten mit Schädigung des ZNS. Ein wichtiger Parameter zur Charakterisierung neuronaler Verarbeitung mittels nichtinvasiver Verfahren ist die Größe und Ausdehnung der kortikalen Aktivierung, was auch als Veränderung kortikaler Karten interpretiert wird. Verbesserte sich durch repetitive sensorische Stimulation der Tastsinn der Finger, waren im somatosensorischen Kortex die Hirngebiete, die taktile Informationen im Finger-/Handbereich verarbeiten, vergrößert ( $\mathbf{A} \mathbf{A b}$. 1). Dies deutet darauf hin, dass zusätzliche Ressourcen rekrutiert wurden, um die Signale aus dem Handbereich effektiver zu verarbeiten. Wenn dies stimmt, sollte die Modifikation der Hirnkarten kausal mit der veränderten Diskriminationsfähigkeit zusammenhängen. Tatsächlich zeigen Probanden, bei denen sich diese Fähigkeit nur gering verbessert, auch nur eine geringe Veränderung der Hirnkarten. Umgekehrt findet bei den Teilnehmern, bei denen sich die Hirnkarten am stärksten verändern, auch die größte Verbesserung des Tastsinns statt [20].

Doppelpuls-Stimulationstechniken Seit einiger Zeit steht die Untersuchung exzitatorischer und inhibitorischer Effekte auf kortikale Erregbarkeit mittels Doppelpuls-Stimulationstechniken im Mittelpunkt vieler Studien. Das Doppelpulsverhalten (paired pulse behavior) ist dadurch gekennzeichnet, dass bei kurzen Interstimulusintervallen bei gleicher Reizstärke die zweite Reizantwort signifikant kleiner ist als die erste. Nach repetitiver sensorischer Stimulation war die Doppelpulssuppression abgeschwächt, wobei der Grad der Suppression positiv mit dem individuellen Zuwachs der Wahrnehmungsleistung korreliert [14].

Konnektivitätsanalysen Ein umfassendes Bild neuronaler Plastizität verlangt neben der Betrachtung lokaler Verarbeitungseigenschaften auch die Analyse der Reorganisation globaler Prozesse, wie dies beispielsweise durch Konnektivitätsanalysen auf der Basis von MR- oder EEG- 
Signalen möglich ist. Untersuchungen der sog. funktionellen Konnektivität mittels EEG oder MR durch Messung des „resting states“ zeigten, dass es nach repetitiver sensorischer Stimulation zu einer Erhöhung der Interaktion in den sensomotorischen Netzwerken kommt [13]. Neuere Untersuchungen haben sogar dafür Hinweise geliefert, dass es im Bereich des somatosensorischen Repräsentationsgebiets nach nur 40 min Stimulation zu strukturellen Veränderungen der grauen Substanz kommt [25].

Diese Befunde zeigen, dass die Gesamtheit der sensomotorischen neuronalen Verarbeitung durch repetitive sensorische Stimulation nachhaltig verändert wird. Es erscheint plausibel, dass diese Signaturen die Grundlage der vielfältigen Verhaltensänderungen bildet.

\section{Generalisierung sensomotorischer Verbesserungen}

Das Training einer bestimmten Aufgabe verbessert diese, allerdings sind solche Verbesserungen immer spezifisch für die trainierte Aufgabe. Vor dem Hintergrund möglicher potenzieller Einsätze als Intervention wird gegenwärtig untersucht, diese oft als „Fluch der Spezifität“ bezeichnete Eigenheit zu überwinden, um Trainingsergebnisse für Alltagssituationen zu generalisieren.

Gezielte Auslösung synaptischer Plastizität Verbesserungen des Verhaltens und der Wahrnehmung werden bei der repetitiven Stimulation nicht durch Training einer Aufgabe erzeugt, sondern durch die gezielte Modifikation synaptischer Übertragung in neuronalen Netzwerken. Daher wurde die Hypothese aufgestellt, dass passive Stimulation alle neuronalen Prozesses umgestaltet, die mit taktiler, haptischer und sensomotorischer Informationsverarbeitung zu tun haben. Eine sich daraus ergebende Vorhersage ist die, dass repetitive Stimulation nicht nur die taktile Diskriminationsfähigkeit verändert, die in vielen Studien verwendet wird.

Generalisierung positiver Effekte In einer Serie von Untersuchungen konnte diese Ausgangshypothese bestätigt werden. So verbesserte sich die taktile Diskriminationsfähigkeit, die Frequenzdiskrimination, Punkt-Muster-Diskrimination, haptische Objektwahrnehmung, Reaktionszeiten bis hin zu sensomotorischem Verhalten, wie beispielsweise Fingergeschicklichkeit [10]. Diese breite Generalisierung positiver Effekte macht den Einsatz der repetitiven Stimulation naturgemäß besonders geeignet für die Therapie und Intervention nach Hirnschädigungen.

\section{Pharmakologische Grundlagen}

Zelluläre Plastizitätsstudien legen nahe, dass nur wenige fundamentale Mechanismen die synaptische Übertragung kontrollieren.
NMDA So spielt der N-Methyl-D-Aspartat-Rezeptor (NMDA-Rezeptor) eine zentrale Rolle bei der Regulation synaptischer Plastizität [1][19]. Um zu zeigen, dass auch die Effekte der repetitiven Stimulation solchen plastizitätsvermittelnden Mechanismen unterliegen, wurde die Abhängigkeit der repetitiven Stimulation von NMDA-Rezeptoren untersucht. Dazu erhielten Versuchspersonen eine einmalige Gabe von Memantin, einer Substanz, die selektiv NMDA-Rezeptoren blockiert. In dieser placebokontrollierten Studie zeigte sich, dass Memantin den Lernerfolg nach repetitiver Stimulation vollständig blockierte, und zwar sowohl auf perzeptueller als auch auf kortikaler Ebene [7].

GABA Ein weiterer zentraler „Player“ ist GABA. GABA spielt eine wichtige Rolle in der Aufrechterhaltung der Balance zwischen Erregung und Inhibition und ist dadurch bei allen Verarbeitungsprozessen sowie bei deren Änderungen aufgrund von Lernen beteiligt. Beim Menschen kann die Rolle von GABA durch Applikation von Medikamenten erfolgen, die GABA-Agonisten enthalten. Nach Gabe einer Einzeldosis eines solchen Medikaments (Lorazepam) wird der typischerweise auftretende Lernerfolg in Form einer Verbesserung der Tastleistung vollständig blockiert [8]. Diese Untersuchungen unterstützen die Annahme, dass repetitive Stimulation synaptische Plastizität auslöst, die durch glutamaterge und GABAerge Rezeptoren kontrolliert wird.

Amphetamin Im Gegensatz zu Ansätzen, plastische Prozesse pharmakologisch zu blockieren, gibt es wenige Möglichkeiten, kortikale Plastizität pharmakologisch zu verstärken. So wird beispielsweise die Auslösung von LTP durch adrenerge Substanzen moduliert. Aus diesem Grund wurden einmalige Gaben von Amphetamin genutzt, um Lernprozesse beim Menschen, die durch repetitive Stimulation hervorgerufen wurden, zu verstärken. Es zeigt sich, dass nach Amphetamin-Gabe die typischen Veränderungen sowohl der taktilen Wahrnehmung als auch der kortikalen Reorganisation nahezu verdoppelt waren [7]. Diese Befunde zeigen, dass die Prozesse, die repetitiver Stimulation zugrunde liegen, durch neuromodulatorische Systeme weiter verstärkt werden können.

\section{Bidirektionale Veränderungen sind frequenzabhängig}

Um die Relevanz von LTP- und LTD-Mechanismen für Verhaltensänderungen beim Menschen zu untersuchen, wurden diese in taktile hoch- oder niedrigfrequente Reizfolgen (tHFS und tLFS) übersetzt. Diese wurden dann als taktile oder elektrische Pulsfolgen auf die Finger übertragen. tHFS bestand aus kurzen Pulsfolgen von jeweils $1 \mathrm{~s}$ Dauer, in denen die Einzelpulse mit $20 \mathrm{~Hz}$ appliziert wurden. Das Intervall zwischen den Pulsfolgen betrug $5 \mathrm{~s}$, tLFS bestand aus einer Serie von Einzelpulsen, die mit $1 \mathrm{~Hz}$ appliziert wurden. Beide Protokolle wurden jeweils für 20 min appliziert. Bereits 20 min nach einer hochfrequenten Stimu- 
lation waren die Diskriminationsschwellen signifikant erniedrigt. Umgekehrt führte tLFS im gleichen Zeitraum zu einer Beeinträchtigung der Diskriminationsfähigkeit [23].

Diese Ergebnisse zeigen, dass die kurze Applikation (<30 min) von Stimulationsprotokollen, die denen der zellulär verwendeten LTP- und LTD-Studien analog sind, verhaltensrelevante und dauerhafte, frequenzabhängige und bidirektionale Veränderungen der menschlichen Wahrnehmung hervorruft. Vor dem Hintergrund der oben skizzierten Uneinheitlichkeit der bisher verwendeten Stimulationsprotokolle (niederfrequent/hochfrequent, kontinuierlich/intermittierend) hat die Bidirektionalität der Effekte erhebliche Relevanz und muss beim Einsatz als Intervention entsprechend berücksichtigt werden.

\section{Prädiktion plastischer Prozesse}

Es ist eine Alltagserfahrung, dass es gute und schlechte Lerner gibt. Dies gilt in gleicher Weise unter Labor- oder klinischen Bedingungen. Warum das so ist, ist weitgehend unklar. Schlechtes Lernen kann viele Ursachen haben: Beeinträchtigungen der Sensorik, sodass bereits beim Aufnehmen des Lernstoffs Probleme entstehen, oder mangelnde Aufmerksamkeit. Es können aber auch Defizite plastischer Mechanismen vorliegen. Ein Beispiel dafür ist der BDNF-Polymorphismus. Kürzlich konnte gezeigt werden, dass auch die Balance der Inhibition und Exzitation einen starken Einfluss auf plastische Prozesse hat. So sagt die mithilfe von MR-Spektroskopie gemessene GABAKonzentration im sensomotorischen Kortex mehr als $50 \%$ einer perzeptuellen Lernaufgabe voraus [12].

Mehr als ein Drittel des Lernerfolgs konnte durch die vor der Induktion von Plastizität durch repetitive Stimulation gemessene Power der somatosensorischen Alpha-Oszillationen (Mu-Rhythmus) vorhergesagt werden [11]. Demnach spielen auch „brain states“ vor und während der Stimulation eine wichtige Rolle. Besonders relevant für klinische Interventionen ist die Möglichkeit, somatosensorische Alpha-Oszillationen durch Neurofeedbacktraining zu verändern, was es ermöglicht, den anschließenden Lernausgang entscheidend zu modulieren [2].

\section{Studien an Patienten}

Metastudie Eine kürzlich veröffentlichte Metastudie basierend auf vier Studien an chronisch durch Schlaganfall betroffenen Patienten (insgesamt 69 Patienten, Zeitraum bis zum Infarkt zehn Monate bis fünf Jahre) berichtet signifikante Verbesserungen der Motorperformanz der oberen Extremität (standardisierte mittlere Differenz, SMD: 1,04; $95 \% \mathrm{KI}: 0,66 \ldots 1,42)$. Als Outcome Measure wurden der Action-Research-Arm-Test, der Jebsen-Taylor-Test, Handkraft und das Fugl-Meyer-Assessment verwendet.
Die Stimulation wurde in zwei Studien als „stand-alone“ durchgeführt, in den zwei anderen jeweils vor dem Training motorischer Aufgaben [6]. In allen Fällen wurde der Medianusnerv stimuliert. Die Stimulation bestand aus Pulsfolgen (Einzelpuls $1 \mathrm{~ms}$, der Tastgrad, „duty cycle“, war 500 ms an, 500 ms aus, Pulsfrequenz $10 \mathrm{~Hz}$ ), die Stimulationsdauer war zwei Stunden. Die Kontrollstimulation bestand entweder in unterschwelliger Stimulation oder in keiner Stimulation („wait group“). In zwei Studien bestand die gesamte Stimulation nur aus einer Session, zwei Studien verwendeten eine mehrfache Applikation über zehn Tage hinweg. Negative Nebeneffekte wurden grundsätzlich nicht beobachtet. Die Autoren der Metastudie schließen daraus, dass repetitive Stimulation ein sicheres Verfahren ist, um als zusätzliche Behandlungsmethode bei chronischen Schlaganfallpatienten zur Behandlung motorischer Defizite der oberen Extremität eingesetzt werden zu können [6].

Zusätzlich verglichen die Autoren der Metastudie die Effektstärke nach Behandlung mit repetitiver Stimulation mit der nach rTMS oder tDCS. Sie berichten, dass diese größer ist (SMD: 1,4; $95 \% \mathrm{KI}: 0,66 \ldots 1,42)$ als die nach tDCS (SMD: 0,45; $95 \%$ KI: 0,09...0,80) oder rTMS (SMD: 0,55; $95 \% \mathrm{KI}: 0,37 \ldots 0,72)$. Dazu weisen sie darauf hin, dass andere Studien keine signifikanten positiven Effekte für rTMS gefunden haben.

Randomisierte klinische Studie Nach Erscheinen dieser Metastudie wurde eine randomisierte klinische Studie an subakut durch Schlaganfall betroffenen Patienten publiziert, bei der ebenfalls die repetitive Stimulation zum Einsatz kam [16]. In dieser Studie wurden insgesamt 46 Patienten untersucht (23 Verum, 23 Placebostimulation, Zeitraum bis zum Infarkt 3-4 Wochen). Die Stimulation bestand aus Pulsfolgen, $1,4 \mathrm{~s}$ an, $5 \mathrm{~s}$ aus, Pulsfrequenz $20 \mathrm{~Hz}$ für 45 min, 10 Tage, mittlere Stromstärke 10,4 mA. Die Stimulation wurde mithilfe eines speziell angefertigten Handschuhs übertragen, der über innenliegende Elektrodenkontakte eine Stimulation der Fingerspitzen aller fünf Finger der betroffenen Hand ermöglichte. Um die unterschiedlichen Schwellen der medianus- und ulnarisinnervierten Finger zu berücksichtigen, konnten diese getrennt angesteuert werden.

Die Kontrollstimulation bestand in unterschwelliger Stimulation, beide Gruppen erhielten Ergo- und Physiotherapie und spezielles Hand-Arm-Training, allerdings ohne feste zeitliche Kopplung an die Stimulation. Die Beurteilung der sensomotorischen Performanz erfolgte durch ein umfangreiches quantitatives Assessment, bei dem die Berührungsschwelle, die räumliche Diskriminationsschwelle, eine 9-Hole-Pegboard-Aufgabe, Handkraft, propriozeptive Aufgaben und ausgewählte Tests aus dem Jebsen-Taylor-Testrepertoire getestet wurden. Die Performanz jeder Einzelaufgabe wurde anschließend in einen Leistungsindex umgerechnet. 
Nach der zweiwöchigen Behandlungsdauer zeigte sich für die Gruppe mit der Kombinationstherapie (Standardtherapie plus repetitive Stimulation) eine signifikant bessere Erholung der gesamten sensomotorischen Performanz (SMD: 0,57; $95 \%$ KI -0,013...1,16; p = 0,027). Auch bei Betrachtung der einzelnen Tests lag die Effektstärke für die Kombinationstherapie jeweils über der der Kontrollgruppe [16].

Untersuchungen und Pilotstudien Zusätzlich zu diesen hier ausführlicher dargestellten Studien liegen zahlreiche Untersuchungen und Pilotstudien vor, die an chronischen oder subakuten Patienten positive Effekte nach Behandlung mit repetitiver Stimulation zeigen, wobei die Stimulation entweder als „stand-alone“ oder als begleitende Intervention genutzt wurde (z. B. [15][18][24]). Da die bisherigen Fallzahlen immer noch klein sind, sind weitere kontrollierte klinische Studien und Multicenter-Studien nötig, um ein tieferes Verständnis für die Wirksamkeit der repetitiven Stimulation bei Patienten mit zentralnervösen Schädigungen zu erhalten.

\section{Ausblick}

Der Ansatz der repetitiven sensorischen Stimulation wurde bisher vor allem an der oberen Extremität bei gesunden Probanden systematisch untersucht. Die dort erhobenen Befunde deuten darauf hin, dass die repetitive sensorische Stimulation zu einer weitreichenden Reorganisation der sensorischen Verarbeitung durch Erhöhung der Interaktion in den sensomotorischen Netzwerken führt. Dies führt zu deutlichen Verbesserungen des Tastsinns, der Propriozeption, der haptischen und motorischen Performanz, was sich seinerseits in einer erhöhten Alltagskompetenz niederschlägt. Daten einer randomisierten klinischen Studie, einer Metastudie sowie Daten zahlreicher Pilotstudien an Patienten sind vielversprechend. Inwieweit die oben genannten Befunde an Gesunden auf Patienten übertragbar sind, ist zurzeit Gegenstand wissenschaftlicher Untersuchung.

Neben dem Aspekt geringer Kosten liegt ein besonderer Vorteil der repetitiven sensorischen Stimulation in der passiven Natur: Es erfordert keine aktive Teilnahme oder besondere Aufmerksamkeit der Teilnehmer. Es ist daher möglich, die Stimulation während anderer Beschäftigungen wie beispielsweise Fernsehen oder Lesen anzuwenden, was die Akzeptanz dieses Verfahrens naturgemäß erhöht und niedrige Abbruchraten zur Folge hat. Die Möglichkeit, die Stimulation zu Hause über lange Zeit hinweg anwenden zu können, spielt für chronische Patienten eine wichtige Rolle.

Damit diese Vorteile in der breiten wissenschaftlichen und nichtwissenschaftlichen Öffentlichkeit Beachtung finden, sind breit angelegte systematische Untersuchungen notwendig. Dabei sind besonders große Fallzahlen und nach Möglichkeit Multicenter-Studien notwendig. Um eine bessere Vergleichbarkeit zwischen den Studien zu erreichen, benötigt man Standards für die einheitliche Anwendung und das Assessment. Weitere Studien sind auch für eine Optimierung der Stimulation hinsichtlich Parameter, Dauer und Stimulationspausen erforderlich. Interessant wäre die Kombination mit der Applikation von Pharmaka. Wenig untersucht sind die Rolle längerer Therapie- und Behandlungszeiten sowie die Auswirkung der Stimulationstherapie lange Zeit nach Beendigung der Behandlung.

Viele Studien schließen Patienten mit nur mäßiger Schwere der Beeinträchtigung ein. Daher wären Studien mit stärker betroffenen Patienten wichtig, um den etwaigen Nutzen der Stimulationstherapie für diese Patientengruppen abschätzen zu können. Vor dem Hintergrund „individualisierter" Therapiemaßnahmen [21] sind weiterführende Studien notwendig, um unsere Kenntnisse über Faktoren und Bedingungen zu erweitern, die Plastizitätsprozesse begünstigen oder erschweren.

\section{Autorinnen/Autoren}

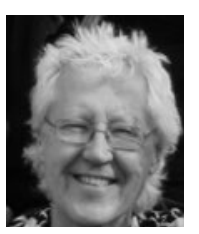

PD Dr. rer. nat. Hubert R. Dinse

Studierte Biologie und Chemie in Mainz und Marburg, promovierte und habilitierte im Fach Zoologie. Er war Postdoc an der Universität Pisa, Italien, freier Mitarbeiter am Battelle-Institut und arbeitete zwei Jahre als Visiting Professor an der University of California in San Francisco (UCSF). Seit 1990 ist er Leiter des von ihm gegründeten Neural Plasticity Lab und Senior Scientist an der Neurologischen Klinik Bergmannsheil. Seine Forschungsschwerpunkte sind Lernen, Alterung, taktile Wahrnehmung sowie die Entwicklung neuer Lern- und Interventionsformen in der Rehabilitation.

\section{Korrespondenzadresse}
PD Dr. rer. nat. Hubert R. Dinse
Neurologische Universitätsklinik und Poliklinik
Berufsgenossenschaftliches Universitätsklinikum
Bergmannsheil
Bürkle de la Camp Platz 1
44789 Bochum
E-Mail: Hubert.Dinse@rub.de

Literatur

Die komplette Literaturliste finden Sie unter www.

thieme-connect.de/products

Bibliografie

DOI https://doi.org/10.1055/a-0754-3281

neuroreha 2018; 10: 178-183

(c) Georg Thieme Verlag KG Stuttgart - New York

ISSN 1611-6496 